\title{
ESTUDIOS DE MÚSICA EN LOS CONSERVATORIOS SUPERIORES Y ANSIEDAD ESCÉNICA EN ESPAÑA
}

\author{
Francisco Javier Zarza Alzugaray, Óscar Casanova López \& Santos Orejudo Hernández
}

\author{
Universidad de Zaragoza \\ fjzarza@unizar.es, ocasanov@unizar.es, sorejudo@unizar.es
}

\begin{abstract}
La ansiedad escénica en músicos en formación es uno de los principales problemas a los que este grupo poblacional debe hacer frente en su práctica diaria. En lo que respecta al estudio de esta problemática en el ámbito formativo español apenas ha recibido atención desde la comunidad investigadora. Con una muestra de 479 estudiantes de Grado Superior de Música en centros españoles, alrededor del 39\% de la población alcanza niveles de ansiedad superiores a la media teórica según el cuestionario diseñado específicamente para evaluar la ansiedad escénica en músicos. Creemos que se hace necesaria una reflexión pedagógica para implementar planes formativos curriculares adecuados encaminados a reducir estas tasas de ansiedad escénica.
\end{abstract}

Palabras clave: Ansiedad escénica, estudiantes, estudios superiores de música, conservatorios de música

Music Performance Anxiety (MPA) in musicians who are undergoing training is one of the most relevant aspects in the daily music practice and performance process. Regarding the study of this problem in the Spanish context, there is a lack of research coming from the scientific community. In this study with a sample of 479 tertiary students of High Degree in music we found that about the $39 \%$ reported anxiety levels over the theoretical mean of the questionnaire designed for the specific purpose of assessing the MPA. We think this result makes necessary a pedagogical reflection about the curriculum towards the reduction of the anxiety levels.

Keywords: Performance anxiety, students, higher degree in music, music conservatories

\section{INTRODUCCIÓN}

\section{Problema en profesionales y estudiantes}

La ansiedad escénica en músicos intérpretes es un problema real al cual una gran parte de ellos deben hacerle frente.

Llegado el momento de exposición pública de las personas y teniendo en cuenta la dimensión temporal del problema, las personas y los músicos en este caso, pueden presentar dificultades cognitivas como fallos de memoria o incapacidad de razonar correctamente, así como pensamientos negativos sobre la actuación o sobre sí mismos. Pueden presentar también e incluso al mismo tiempo que los problemas cognitivos, problemas fisiológicos como palmas de manos con hipersudoración, temblores de manos, brazos, piernas y rodillas, boca seca, temblor de voz, náuseas o incluso vómitos, entre otros. Las personas con problemas de ansiedad escénica pueden también presentar dificultades conductuales como la presencia de conductas de evitación o escape y se puede pensar, tal y como señalan diversos autores, que en función de la intensidad de los síntomas descritos anteriormente y de las interferencias que causen en la actuación, sea necesario algún tipo de tratamiento específico que pueda ayudar a la reducción del problema (LEE, 2002; MCALLISTER, 2011; Petrovich, 2004; SANDGREN, 2002; SPAHN, 2006; TABORSKY, 2007).

Existen estudios que ponen de manifiesto que la ansiedad escénica ocurre normalmente como un desorden relativamente aislado que afecta únicamente a una parte muy específica de la vida de la persona que la padece (KENNY \& ACKERMANN, 2009), encontrando este mismo panorama entre los profesionales de la música (VAN KEMENADE, VAN SON \& VAN HEESCH, 1995). Además de tocando solos, la ansiedad escénica en los músicos es sufrida por éstos también en conciertos con amigos o interpretando solos ante los profesores o colegas de profesión, y aunque se puede afirmar que la mayoría de los músicos suelen sufrir cierto grado de ansiedad escénica, no suelen sufrir problemas de ansiedad generalizada (CLARK \& AGRAS, 1991).

Según Ortiz Brugués (2008), y continuando con datos de incidencia de este problema en la población musical, encontramos que el $80 \%$ de la misma sufre ansiedad escénica cuando se les 
requiere actuar frente a una audiencia. Sin embargo, en una minoría poblacional pueden presentarse desordenes co-mórbidos siendo el más común el trastorno de ansiedad generalizada y aprensión en múltiples facetas de la vida y no solamente en las situaciones que requieran una actuación pública.

Estudios como el de Fishbein, Middlestadt, Ottati, Straus y Ellis (1988) sitúan a la ansiedad escénica como el problema no musco-esquelético más frecuente y en el que al menos el $16 \%$ de los músicos profesionales consultados indicaba que la ansiedad escénica presenta un problema real con efectos muy negativos en sus actuaciones.

En cuanto a las condiciones de trabajo y estudio de los músicos profesionales, Sternbach (1995, 2008), describió las condiciones de éstos como generadoras de una tasa de estrés total que excede por mucho al observado en otras profesiones, pudiendo generar, tal y como señala Chan (2011), en diferentes problemas en las actuaciones y problemas psicológicos más severos.

En España la población actual de músicos en formación superior apenas ha recibido la atención merecida tanto en el ámbito pedagógico como en el investigador y en opinión de autores como Herrera y Jorge (2013) o Zarza, Casanova y Orejudo (2016a), el estudio de este problema es relativamente reciente en la literatura científica.

Debemos señalar que la ansiedad escénica es un problema presente en otros contextos formativos (OREJUDO, FERNÁNDEZ \& BRIZ, 2012) y en lo relacionado con la práctica musical hay estudios que ponen de manifiesto que en torno a un $20 \%$ de la población abandona sus estudios debido a la ansiedad escénica y de los que continúan, entre el $40 \%$ y el $60 \%$ ven empeoradas significativamente sus actuaciones públicas (DALIA, 2004; MARCHANT-HAYCOX \& WILSON, 1992).

Investigaciones como las de Kaspersen y Götestam (2002) concluyeron que en torno al 36.5\% de los estudiantes $(n=126)$ significaron la necesidad de ayuda para la solución de problemas asociados a la ansiedad escénica. Además Fehm y Hille (2005) encontraron que el $40 \%$ de los 103 estudiantes estudiados, sufría estrés a causa de la ansiedad escénica y que un $20 \%$ veía empeorada la calidad de su actuación debido a este problema. También estudios como el de Chan (2011) muestran cómo la ansiedad es el problema más común entre los estudiantes de música alcanzando una prevalencia del $65.5 \%$ siendo el miedo al fallo el principal motivo causante de la ansiedad escénica. En este sentido estudios como el de Tamborrino (2001) ponen de manifiesto que el $91.7 \%$ de los estudiantes había manifestado ansiedad escénica alguna vez en su vida inmediatamente antes de la actuación, un $86.5 \%$ durante la misma y un 76.5\% informaban que la ansiedad escénica había afectado negativamente a sus actuaciones. Asimismo, se constata que existe el problema con independencia del centro de enseñanza musical (ZARZA ET AL., 2016a).

En lo concerniente a los planes formativos y normativos de la enseñanza de Grado Superior de Música en España, ésta se encuentra en un proceso de remodelación, tanto en lo que hace referencia a sus prácticas, como al análisis de los perfiles profesionales o al entorno legislativo en el que se mueve. La parte legislativa ha de atender a diferentes aspectos como los relacionados con el perfil profesional, la mejora de las prácticas pedagógicas o los sistemas de evaluación. En este sentido encontramos necesario dedicar un breve apartado a la explicación del marco legal por el que se han venido rigiendo, en los últimos años, las enseñanzas superiores en los conservatorios de música en España.

\section{Concepto de ansiedad escénica}

Salmon (1990) definió la ansiedad escénica como la experiencia de aprensión angustiosa sobre la disminución real de las habilidades interpretativas en un contexto público, hasta un grado que no garantice la aptitud musical del individuo, el entrenamiento o el nivel de preparación de éste.

Hoy en día, en la comunidad científica existe un consenso bastante amplio en que la ansiedad escénica está caracterizada por una evidente y perceptible aprensión a la actuación (KENNY, 2008; 
Salmon, 1990; Studer, Danusera, Hildebrand, Arial \& Gomez, 2011; Wilson, 1997) y que ésta presenta tres componentes claramente diferenciados conocidos habitualmente como cognitivo, fisiológico y conductual (OSBORNE, KENNY \& HOLSOMBACK, 2005; ZARZA ET AL., 2016a).

El modelo que desde esta investigación se ha tenido en cuenta para explicar la génesis y mantenimiento de los problemas de ansiedad en la población de estudiantes de Grado Superior de Música de España ha sido el modelo de ansiedad descrito por Barlow (2000) y operativizado posteriormente por Kenny, Davis y Oates (2004) y Zarza et al. (2016a). En dicho modelo Barlow expone que la ansiedad puede explicarse a partir de tres factores o vulnerabilidades generadores de la misma. Encontramos un primer factor, en cierto modo heredable, que hace referencia a la existencia de una vulnerabilidad biológica y en la que distintos factores biológicos difíciles de localizar contribuyen en la posible génesis de distintos desórdenes de ansiedad y afectividad negativa. En segundo lugar encontramos la presencia de una vulnerabilidad psicológica general, que tiene como base las experiencias tempranas y la falta de control personal de los eventos del entorno más cercano. Debemos observar que relacionado con este factor de vulnerabilidad general, las experiencias adversas en edades tempranas acaecidas en determinadas condiciones contribuyen a incrementar la denominada "vulnerabilidad psicológica" y a experimentar ansiedad y afectividad negativa como estado emocional general. El tercer factor de vulnerabilidad que Barlow describe en su modelo es el denominado como "vulnerabilidad psicológica específica". Dicho factor está determinado por los estímulos ambientales concretos, los diferentes tipos de aprendizaje, condicionado o vicario y por ende, toman mayor importancia las experiencias directamente relacionadas con la actuación. Este último factor resulta una condición necesaria para la presencia de ansiedad y a la postre, la conjunción de todos estos factores determinan la presencia de mayores o menores niveles de ansiedad escénica o incluso de la génesis de desórdenes específicos de ansiedad como las fobias específicas o la fobia social (BARLOW, 2000; KENNY ET AL., 2004; KENNY \& OSBORNE, 2006).

\section{LEGISLACIÓN SOBRE ENSEÑANZA MUSICAL SUPERIOR}

Alberto Veintimilla en el informe sobre el estado de las enseñanzas artísticas en España indica que para una adaptación de los planes de estudio nacionales al marco europeo, los estudios superiores de grado de música se pueden distribuir en: "Estudios orientados principalmente a potenciar el desarrollo de la personalidad y la competencia artística del estudiante, estudios orientados principalmente a incrementar las competencias teóricas y de investigación, y estudios orientados a incrementar las competencias artísticas con las teóricas” (2010: p. 146-147).

El modo en el que se legisla parece tener gran importancia en la práctica formativa musical y que por ende, puede afectar también a la ansiedad escénica en los músicos en formación. Este modo de legislar creemos que acarrea consecuencias trascendentales, no solo a nivel nacional sino internacional, más aún si cabe, en el entorno de integración europea en el que el Estado Español está inmerso en numerosos ámbitos, incluido el de la educación.

Para tratar de lograr los criterios de convergencia anteriormente expuestos por Veintimilla (2010), el marco normativo español lleva realizando una serie de reformas en la legislación que regula la educación musical española que pretenden lograr la similitud con el marco común europeo de formación superior.

En la Ley Orgánica 2/2006, de 3 de mayo, de Educación (L.O.E.), se regulan de forma específica, en sus artículos 54 a 58, las enseñanzas artísticas superiores señalando como tales y entre otras a los estudios superiores de música. La finalidad de las mismas es "proporcionar al alumnado una formación artística de calidad y garantizar la cualificación de los futuros profesionales de la música". 
Con posterioridad, el Real Decreto 1614/2009, de 26 de octubre, por el que se establece la ordenación de las enseñanzas artísticas superiores, ordena las enseñanzas artísticas superiores y entre ellas la música, contemplando un planteamiento más global e integrador dentro de un sistema educativo, el español, que busca la consonancia con el Espacio Europeo de la Educación Superior.

Para tratar de conseguir esta integración y asimilación normativa con el marco europeo, la flexibilización de la enseñanza y la renovación de las metodologías constituyen el punto de partida para que se mejoren los procesos de aprendizaje, la adquisición de competencias, la realización de prácticas externas, la movilidad y la promoción del aprendizaje a lo largo de la vida por parte de los estudiantes, y la adecuación de los procedimientos de evaluación por parte del profesorado.

Posteriormente, el Real Decreto 631/2010, de 14 de mayo, por el que se regula el contenido básico de las enseñanzas artísticas superiores de Grado en Música, destaca el esfuerzo de adaptar la legislación a un entorno europeo, siendo necesario el cuidado de aspectos tan esenciales en el proceso de aprendizaje musical como son el equilibrio entre los conocimientos conceptuales, el desarrollo de destrezas técnicas y la comprensión de los principios culturales y estéticos que determinan el fenómeno artístico.

Desde el marco teórico esbozado por Veintimilla (2010), y de cara a alcanzar unos conocimientos prácticos que unifiquen todo el territorio europeo, las enseñanzas artísticas superiores se regulan a partir del Real Decreto 1614/2009 y el Real Decreto 631/2010, además de las consiguientes ordenes autonómicas por las que se regulan los contenidos básicos y se establecen los planes de estudios de las enseñanzas artísticas superiores de Grado de Música establecidas en la Ley Orgánica de Educación 2/2006, de 3 de mayo.

Como señalamos, la L.O.E. establece que las enseñanzas superiores de música tienen como primer objetivo general "la formación cualificada de profesionales que dominen los conocimientos propios de la música y adopten las actitudes necesarias que les hagan competentes para integrarse en los distintos ámbitos profesionales de esta disciplina”.

Asimismo, y tal y como se señala en el articulado del mencionado Real Decreto 631/2010, en lo referente a la evaluación del proceso de aprendizaje del alumnado, ésta se debe basar en la adquisición y consolidación de las correspondientes competencias, tanto transversales como generales y específicas, de forma que posea un carácter integrador dentro del corpus de todos los estudios de Grado Superior de Música. Entre las competencias que se mencionan en el antedicho artículo, encontramos algunas que enlazan directamente con la necesidad de estudiar el constructo de la ansiedad escénica para poder ofrecer al alumnado una mejor formación, y que éste pueda "Buscar la excelencia y la calidad en su actividad profesional" (p. 48488) u "Organizar y planificar el trabajo de forma eficiente y motivadora" (p. 48488).

En nuestro caso, la ansiedad escénica enlaza directamente con la posible adquisición de mejor o peor modo de una serie de competencias específicas para los instrumentistas, correspondientes a la especialidad de Interpretación, que se recogen en el anteriormente citado marco legal. Así pues y a modo de ejemplo, algunas normativas autonómicas, como la Orden del Gobierno de Aragón de 14 de septiembre de 2011, la del Gobierno de la Comunidad de Madrid en su Decreto 36/2010, de 2 de junio de 2011, la Resolución de 25 de julio de 2013 de la Consejería de Educación, Universidades y Empleo de la Región de Murcia o el Decreto de la Consejería de Educación de la Comunidad Autónoma de Castilla y León 57/2011 de 21 de septiembre, señalan las competencias y contenidos necesarios para la obtención del título de Grado Superior de Música en los itinerarios instrumentales, especialidad de Interpretación. De todos ellos, se pueden señalar relacionados con la interpretación en público, entre otros, los siguientes objetivos y contenidos:

- Interpretar el repertorio significativo de su especialidad tratando de manera adecuada los aspectos que lo identifican en su diversidad estilística. 
- Demostrar capacidad para interactuar musicalmente en todo tipo de proyectos musicales participativos, desde el dúo hasta los grandes conjuntos.

- Expresarse musicalmente con su instrumento/voz de manera fundamentada en el conocimiento y dominio en la técnica instrumental y corporal; comunicar, como intérprete, las estructuras, ideas y materiales musicales con rigor.

- Argumentar y expresar verbalmente sus puntos de vista sobre la interpretación, así como responder al reto que supone facilitar la comprensión de la obra musical.

- Síntesis y dominio de las dimensiones básicas de la interpretación musical profesional.

- Desarrollo de un estilo propio como intérprete y de madurez creativa así como hábitos y técnicas de estudio y valoración crítica del trabajo.

- Control de correctos hábitos posturales y técnicas de relajación; preparación para la interpretación en público, como solista o junto a otros intérpretes.

- Aprender a controlar de forma consciente las respuestas psicológicas y fisiológicas en situaciones de estrés. Controlar el miedo escénico.

- Conocer las implicaciones escénicas que conlleva su actividad profesional y ser capaz de desarrollar sus aplicaciones prácticas.

- Técnicas de concentración aplicadas al estudio y a la actuación en público para optimizar la preparación del concierto y la interpretación musical en el escenario.

- Desarrollo de un comportamiento correcto y eficaz en el escenario.

- Control del miedo escénico y preparación a la interpretación en público.

Creemos, por todo lo que se lleva expuesto, que el tratamiento normativo de la ansiedad escénica puede influir en la formación de los estudiantes de música en general y particularmente en los de grado superior, pudiendo incluso llegar a convertirse en un problema muy perjudicial a la hora de alcanzar las competencias mínimas que, según la legislación, deben ser adquiridas para la obtención de un título superior de música, y que en el caso de los itinerarios instrumentales de la especialidad de Interpretación se puede concretar en la competencia específica "Conocer las implicaciones escénicas que conlleva su actividad profesional y ser capaz de desarrollar sus aplicaciones prácticas" (Real Decreto 631/2010: p. 48491) o como se define en el perfil profesional de la misma especialidad "Deberá estar preparado para ejercer una labor interpretativa de alto nivel de acuerdo con las características de su modalidad y especialización, tanto en el papel de solista como formando parte de un conjunto, así como, en su caso, en su condición de intérprete acompañante de música y danza" (p. 48494).

\section{METODOLOGÍA}

\section{Objetivos y muestra}

El principal objetivo de esta investigación ha sido analizar la presencia de niveles de ansiedad y perfiles de vulnerabilidad ante la ansiedad en el alumnado intérprete-instrumentista de Grado Superior de Música de España.

Para ello se ha contado con una muestra de 482 estudiantes. Sin embargo, se ha dispuesto de una muestra útil (n) de 479 estudiantes de música (54\% hombres y 46\% mujeres), de grado superior de los Conservatorios Superiores de Música del País Vasco, Región de Murcia, Castilla y León, Principado de Asturias e Islas Canarias, debido a que 3 estudiantes no indicaron ni edad, ni sexo, ni familia instrumental. Respecto a los 479 útiles, encontramos que en todos los ítems la tasa de respuesta a los mismos del cuestionario es superior al 99\%, habiéndose completado los escasos valores perdidos con la media muestral tal y como realizan para estos casos, con tasas de valores perdidos inferiores al 5\%, autores como Pilatti, Godoy y Brussino (2011).

La muestra ha seguido un criterio de disponibilidad sin existencia de muestreo, es decir, se han podido obtener datos de los centros, y los estudiantes, que han querido participar en esta 
investigación, habiéndose solicitado la participación a todos los conservatorios superiores de música españoles. Así, fue un miembro del equipo de investigación quien entre octubre de 2011 y enero de 2012 se desplazó a los centros anteriormente mencionados; en las clases con una mayor ratio de estudiantes, agrupaciones orquestales, banda e historia de la música, les proporcionó un cuestionario diseñado ad hoc para que lo completaran, asegurando en todo momento el anonimato y voluntariedad de los participantes.

La edad media de los participantes es de 22.64 años (23.15 chicos; 22.04 chicas) y en cuanto a las familias instrumentales el $29.2 \%$ son instrumentistas de cuerda frotada, el $27.1 \%$ instrumentistas de viento madera, el $14.8 \%$ instrumentistas de viento metal, el 14.4 instrumentistas de tecla, el $6.5 \%$ instrumentistas de cuerda pulsada, el $4 \%$ de canto, el $2.7 \%$ estudiantes de percusión y un $1.3 \%$ instrumentistas que además están estudiando bien dirección de coro, bien pedagogía o bien dirección orquestal (Figura 1).

En este sentido debemos señalar de cara a la representatividad muestral cómo los porcentajes de chicos y chicas de este estudio son similares a los datos globales ofrecidos por el Ministerio de Educación sobre las personas matriculadas en el grado superior de música del curso 2011/2012 en donde encontramos un $59.1 \%$ de chicos y un $40.9 \%$ de chicas en edades la mayor parte de ellos comprendidas entre los 18 y 24 años al igual de como ocurre en el estudio aquí presentado. Además, no existe asociación significativa ni entre el sexo de los estudiantes y el estudio de un instrumento solista $\mathrm{u}$ orquestal $\left(\chi^{2}=0.206 ; \mathrm{p}=0.650\right) \mathrm{ni}$ del sexo con la edad de los participantes $\left(\chi^{2}=7.001 ; p=0.072\right)$.

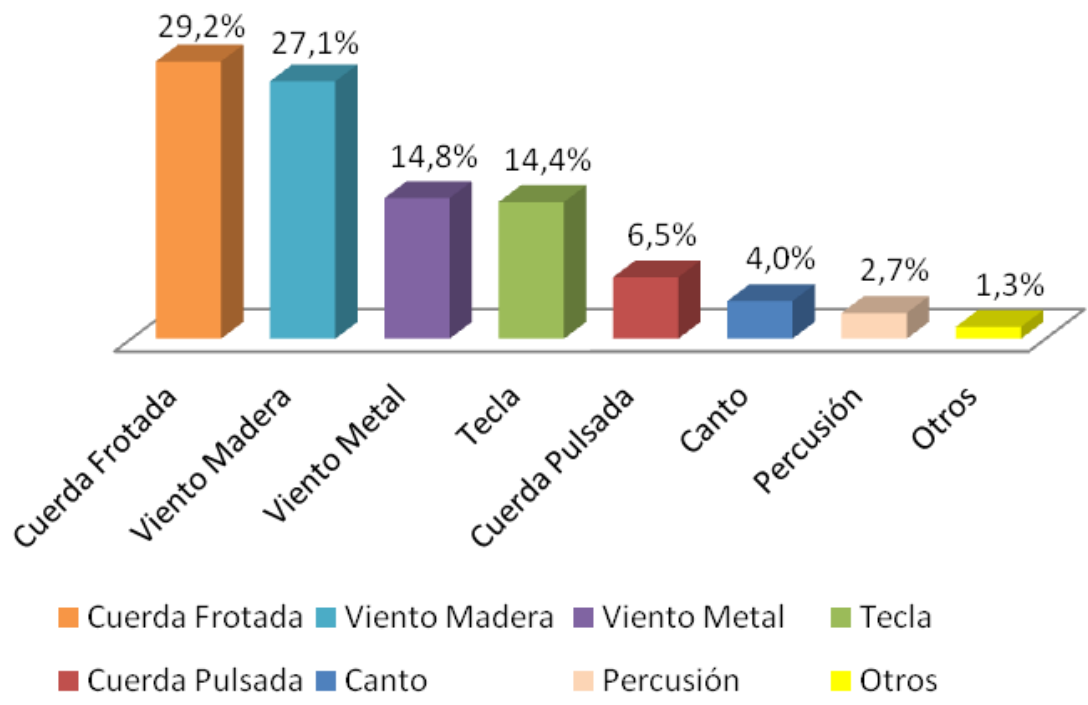

Figura 1. Distribución de las familias instrumentales

\section{Método e instrumento}

La metodología empleada es cuantitativa obteniendo resultados a partir de las respuestas a un cuestionario adaptado ad hoc para la investigación, y el tratamiento estadístico de los datos ha sido realizado con el programa informático SPSS 19. Como se ha mencionado, los datos fueron obtenidos de manera directa por el propio equipo investigador en las clases más numerosas a las que acudían los instrumentistas.

Para este trabajo se ha empleado la adaptación al castellano realizada por el propio equipo de investigación (ZARZA, OREJUDO, CASANOVA \& MAZAS, 2016b) del cuestionario de 2004 denominado Kenny Music Performance Anxiety Inventory, KMPAI, en adelante. El KMPAI es un 
instrumento que fue construido por Kenny, Davis y Oates (2004), y permite evaluar desde el modelo de ansiedad de Barlow (2000) la ansiedad escénica. Existen diferentes versiones del KMPAI en distintos idiomas, y en el proceso de adaptación al castellano (ZARZA ET AL., 2016b), se evaluaron las propiedades psicométricas del cuestionario mostrando un claro encuadre dentro de la aproximación de Barlow y en donde la dimensión de vulnerabilidad general (10 ítems) mostró un alfa de Cronbach igual a 0.786 y la escala para evaluar el contexto de relaciones tempranas un alfa de Cronbach igual a 0.568 (3 ítems); a su vez la escala específica de ansiedad escénica (11 ítems) mostró un alfa de Cronbach igual a 0.868 , muy superior al 0.70 mínimo exigido habitualmente por la comunidad científica para poderla considerar fiable (DE VELLIS, 2003; KLINE, 2000). Así, el cuestionario empleado en esta investigación ha presentado buenas propiedades psicométricas, resultando ser válido para la evaluación de la ansiedad escénica en estudiantes españoles de Grado Superior de Música; esta dimensión específica del cuestionario está compuesta por 11 ítems y presenta 7 opciones de respuesta de acuerdo con una escala de puntuación tipo Likert, siendo la puntuación de 4 puntos la valoración media y mediana (teórica) de la escala, dejando 3 opciones de puntuación mayor y menor a ésta valoración de 4 puntos.

Puntuaciones más elevadas indican mayores niveles de ansiedad escénica mientras que puntuaciones menores indican menores niveles de ansiedad escénica.

En la adaptación al castellano (ZARZA ET AL., 2016b), los enunciados de los 11 ítems que corresponden a las cogniciones específicas de ansiedad son:

1. Cuanto más trabajo en la preparación de un concierto, más probable es que cometa un error importante.

2. Antes de los conciertos no sé nunca si haré una buena interpretación.

3. Durante las interpretaciones, llego a cuestionarme si llegaré al final de la interpretación.

4. Pensar en cómo voy a ser evaluado interfiere con mi interpretación.

5. Incluso durante las ejecuciones más estresantes, confío en que haré una buena interpretación.

6. A menudo me preocupa la reacción negativa del público.

7. Desde el principio de mis estudios, recuerdo tener ansiedad ante las interpretaciones.

8. Me preocupa que una mala interpretación arruine mi carrera.

9. Renuncio a interpretaciones interesantes debido a la ansiedad.

10. A menudo me preparo para un concierto con una sensación de terror y desastre.

11. Me preocupo tanto antes de una interpretación que no puedo dormir.

\section{Resultados}

En este estudio mostramos las relaciones intrínsecas generales de la dimensión específica de ansiedad escénica, así como las diferentes valoraciones emitidas por los estudiantes participantes en el estudio a las preguntas que en ella se recogen.

En lo que respecta a los resultados individuales de los ítems, encontramos tal y como puede comprobarse en la Figura 2 que 5 ítems de los 11 que componen la escala de cogniciones específicas presentan valores mayores de la media teórica del cuestionario. Por una parte, podemos observar que los estudiantes ante las preguntas 7 (Desde el principio de mis estudios, recuerdo tener ansiedad ante las interpretaciones), 2 (Antes de los conciertos no sé nunca si haré una buena interpretación), 6 (A menudo me preocupa la reacción negativa del público) y 4 (Pensar en cómo voy a ser evaluado interfiere con mi interpretación) puntúan más alto que la media teórica (4 puntos), lo que nos indica que son las afirmaciones que más ansiedad les generan.

Por otra parte, encontramos también 6 ítems con puntuaciones más bajas de la media teórica. Así los ítems 8 (Me preocupa que una mala interpretación arruine mi carrera), 11 (Me preocupo tanto antes de una interpretación que no puedo dormir), 10 (A menudo me preparo para un concierto con una sensación de terror y desastre), 3 (Durante las interpretaciones, llego a 
cuestionarme si llegaré al final de la interpretación), 9 (Renuncio a interpretaciones interesantes debido a la ansiedad) y 1 (Cuanto más trabajo en la preparación de un concierto, más probable es que cometa un error importante), son los ítems que presentan puntuaciones menores que la media teórica, siendo además significativamente diferentes a ésta $(\mathrm{p}<0.05)$.

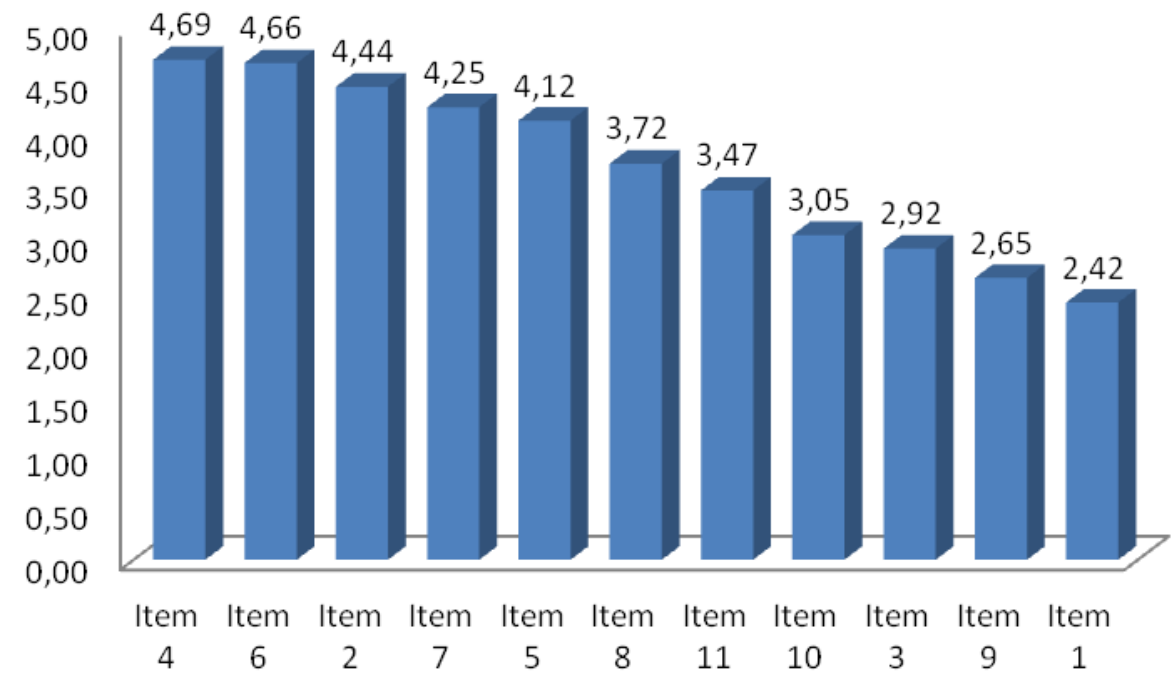

Figura 2. Resultados de los ítems

$\mathrm{Si}$ analizamos las relaciones entre las preguntas mediante un análisis de correlaciones, vemos que éstas son todas significativas $(\mathrm{p}<0.001)$ en todos los casos tal y como se refleja en la Tabla 1.

En términos generales, encontramos un corpus de correlaciones que se podrían calificar como importantes que superan el índice correlacional de 0.3 y en numerosas ocasiones el 0.4 dando muestra de las buenas propiedades psicométricas de la escala utilizada.

Más pormenorizadamente, encontramos resultados en los que esta relación es especialmente relevante ( $r>0.5)$ como la existente entre las preguntas 10 y 2, 3, 9 y 11 o la pregunta 8 con la 7 . Por otra parte, encontramos relaciones, que aún teniendo índices de significación muy bajos menores de 0.001, no presentan valores correlacionales demasiado potentes $(<0.3)$. En esta casuística encontramos las relaciones entre el ítem 1 y los ítems 3, 4, 5, 6, 7, 8, 9 y 11.

\begin{tabular}{|c|c|c|c|c|c|c|c|c|c|c|c|}
\hline & $\begin{array}{c}\text { Ítem } \\
1\end{array}$ & $\begin{array}{c}\text { Ítem } \\
2\end{array}$ & $\begin{array}{c}\text { Ítem } \\
3\end{array}$ & $\begin{array}{c}\text { Ítem } \\
4\end{array}$ & $\begin{array}{c}\text { Ítem } \\
5\end{array}$ & $\begin{array}{c}\text { Ítem } \\
6\end{array}$ & $\begin{array}{c}\text { Ítem } \\
7\end{array}$ & $\begin{array}{c}\text { Ítem } \\
8\end{array}$ & $\begin{array}{c}\text { Ítem } \\
9\end{array}$ & $\begin{array}{c}\text { Ítem } \\
10\end{array}$ & $\begin{array}{c}\text { Ítem } \\
11\end{array}$ \\
\hline Ítem 1 & 1 & .348 & .266 & .195 & .208 & .188 & .178 & .276 & .287 & .380 & .248 \\
\hline Ítem 2 & .348 & 1 & .391 & .362 & .453 & .380 & .414 & .354 & .311 & .533 & .387 \\
\hline Ítem 3 & .266 & .391 & 1 & .338 & .381 & .307 & .304 & .349 & .345 & .528 & .358 \\
\hline Ítem 4 & .195 & .362 & .338 & 1 & .417 & .386 & .419 & .428 & .341 & .422 & .323 \\
\hline Ítem 5 & .208 & .453 & .381 & .417 & 1 & .329 & .405 & .332 & .390 & .494 & .368 \\
\hline Ítem 6 & .188 & .380 & .307 & .386 & .329 & 1 & .341 & .482 & .329 & .438 & .371 \\
\hline Ítem 7 & .178 & .414 & .304 & .419 & .405 & .341 & 1 & .508 & .308 & .479 & .460 \\
\hline Ítem 8 & .276 & .354 & .349 & .428 & .332 & .482 & .508 & 1 & .318 & .471 & .409 \\
\hline Ítem 9 & .287 & .311 & .345 & .341 & .390 & .329 & .308 & .318 & 1 & .532 & .409 \\
\hline Ítem 10 & .380 & .533 & .528 & .422 & .494 & .438 & .479 & .471 & .532 & 1 & .525 \\
\hline Ítem 11 & .248 & .387 & .358 & .323 & .368 & .371 & .460 & .409 & .409 & .525 & 1 \\
\hline
\end{tabular}

** La correlación es significativa al nivel 0.01 (bilateral).

Tabla 1. Correlaciones entre ítems** 
En lo que respecta a los resultados de la escala, habiendo hecho el sumatorio correspondiente de los ítems anteriores, debemos señalar que para la muestra de estudiantes de Grado Superior de Música de los centros que colaboraron con la investigación, la media obtenida es igual a 40.359 $(\mathrm{DT}=12.776)$ valor éste muy cercano a la media teórica del cuestionario que es 44.0. Además, los índices de asimetría (0.41) y de curtosis (-1.951) indican un buen ajuste de la distribución de los datos con una curva normal.

A tenor del análisis de las respuestas encontramos también que alrededor del $39 \%$ de la población presenta niveles de ansiedad escénica por encima de la media teórica, tal y como puede verse en la siguiente figura (Figura 3):

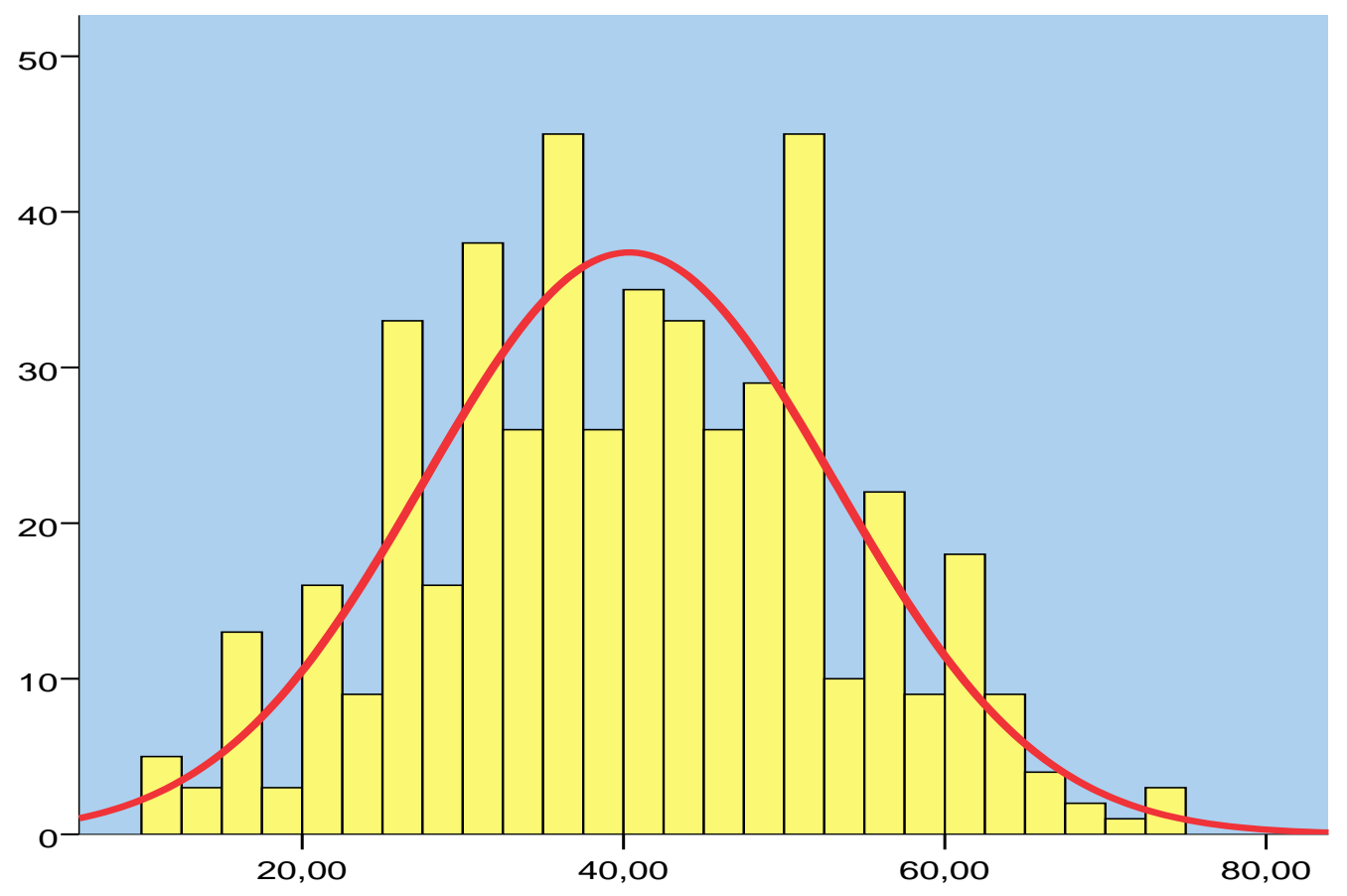

Figura 3. Distribución general de casos en Factor Específico de Ansiedad Escénica

Además de lo anterior, analizando las respuestas de los estudiantes hemos comprobado que la moda de respuestas se sitúa en 43 puntos (3 puntos por encima de la media) y que existe aproximadamente un $15 \%$ de la población con puntuaciones mayores que una desviación típica respecto de la media.

\section{CONCLUSIONES}

A tenor de los resultados expuestos anteriormente, podemos concluir que la ansiedad escénica es un problema presente en la población de estudiantes superiores de música españoles de manera muy importante y que está presente en porcentajes muy similares a los encontrados en otras investigaciones como las de Kaspersen y Götestam (2002) con un 36.5\% de prevalencia, Fishbein et al. (1988) con un $37 \%$ o Fehm y Hille (2005) con un $40 \%$.

Se constata que un $15 \%$ de la población estudiada supera con creces (más de una desviación típica) la media teórica. Se podría pensar que precisamente sean estos estudiantes los que mayor atención necesiten de cara a evitar cuadros clínicos o subclínicos de problemas de ansiedad. 
También pueden ser los que más probabilidades tengan de abandonar los estudios musicales por este problema, porcentaje cercano al 20\% señalado por Dalia (2004).

Con todo lo anterior, debemos tener en cuenta que los aspectos relacionados con la ejecución musical son considerados por la propia normativa como fundamentales para fomentar la creatividad musical y el desarrollo de competencias que faciliten al alumnado un nivel de aprendizaje de acuerdo con las necesidades específicas del ejercicio profesional de la música. Este cambio legislativo con una marcada tendencia profesionalizadora del ejercicio de la música debe tener en cuenta también una reforma en la práctica formativa para que ésta mejore y revierta sus resultados en los futuros profesionales de la música.

Es innegable también que la formación de los alumnos para alcanzar los objetivos que la norma promulga pasa por tener un tejido de profesores cualificados y formados que actúen como facilitadores activos en la adquisición de habilidades técnicas, musicales, investigadoras, etc.

Desde esta investigación se asume que el conocimiento del constructo de la ansiedad escénica por parte del profesorado ayuda de forma indefectible a una formación más cualificada del alumnado. De este mismo modo, también se recoge en el articulado de los contenidos básicos de las enseñanzas del Grado de Música, que éstas deben contemplar "una formación orientada a la preparación para el ejercicio de actividades de carácter profesional" (Real Decreto 1614/2009: p. 89747), y es en esta preparación que indica la legislación donde tradicionalmente se ha obviado el aspecto más específico de la formación para ofrecer conciertos, centrándose prácticamente en exclusiva en la preparación de repertorio y no en la posible defensa del mismo en público, entrando este punto en contradicción con lo que la norma dicta y lo que la realidad de práctica profesional impone.

\section{REPERCUSIONES Y PROSPECTIVA}

Una extensión inmediata al marco normativo expuesto con anterioridad nos lleva a pensar cómo se pueden impartir esa serie de conocimientos y formación que tradicionalmente ha sido dejada al "buen hacer" del profesor. Creemos que las enseñanzas musicales superiores españolas en los conservatorios deben beber del actual paradigma educativo general, y de este modo hacer un trasvase de conocimientos de métodos pedagógicos, contenidos curriculares y objetivos, así como de temporización, de la enseñanza de otros entornos docentes en los que estas cuestiones, que en el marco normativo español están empezándose a contemplar, llevan ya tiempo arrojando resultados positivos (Alsina, 1997). A su vez, también se hace necesaria una mayor formación específicamente pedagógica (BÚJEZ, 2008).

De forma natural, se puede asumir que la investigación en sí misma constituye un elemento crucial de cualquier cultura de aprendizaje, para incorporarla así a la mejora de la práctica docente. Coincidimos con Bisquerra (2000) cuando afirma que las conclusiones en investigación pedagógica deben conducir a unas implicaciones para la práctica educativa. Así pues, siguiendo a Renshaw (1999), encontramos que los centros de enseñanzas artísticas necesitan, de una forma $u$ otra, convertirse en laboratorios artísticos en donde la práctica interpretativa, los procesos creativos y los diferentes modos de aprendizaje se interrelacionen para, asemejando un bucle, puedan desembocar en una mejora docente y en una mejor formación del alumnado.

De este modo, se debería ampliar el marco teórico sobre el origen, desarrollo y mantenimiento de la ansiedad, de tal manera que podamos conocer los factores que inciden en la misma y poder plantear estrategias de intervención, tanto en los procesos formativos como quizás de manera más específica para aquellas personas con mayor afección por el problema. Este tipo de investigaciones contribuyen a una mejora docente y formativa del alumnado; un alumnado que de forma progresiva tendrá que abandonar la formación meramente académica y ocupar la posición de docente, ya que 
es ésta la salida más habitual (CID, 2012), en donde también "estos futuros profesores, tendrán que interpretar frente a sus futuros estudiantes" (YAĞIŞHAN, 2009: p. 9).

Creemos que la no implementación de planes específicos de formación dentro de los centros superiores de música facilita la presencia de niveles de ansiedad elevados en la población de estudiantes de música, haciendo necesaria la creación de planes normativos y formativos específicos que persigan la mejor formación de los futuros profesionales de la música de España.

Relacionado con las ideas precedentes, encontramos que la preocupación por parte tanto del profesorado como del alumnado en lo referido a la necesidad legal y curricular de la existencia de este tipo de formación es real. De hecho, en el estudio de Tamborrino (2001), se muestra cómo un $65 \%$ del profesorado y un $80.3 \%$ del alumnado creen que la prevención y reducción de la ansiedad escénica debería ser contemplada como asignatura en el currículo formativo.

Lo relevante en la reflexión anterior no es sólo que los profesores aprendan a desarrollar estrategias de afrontamiento y superación de la ansiedad escénica a la hora de tocar en público y delante de su alumnado, sino que puedan también enseñar a los estudiantes cómo generar conductas de afrontamiento positivas para tener, en definitiva, mayores garantías de éxito en el aprendizaje de la profesión musical del alumnado.

\section{Referencias}

Alsina, P. (1997). El área de educación musical. Propuestas para aplicar en el aula. Barcelona: Graó.

Barlow, D. H. (2000). Unraveling the mysteries of anxiety and its disorders from the perspective of emotion theory. American Psychologist, 55(11), 1247-1263. DOI: 10.1037/0003066X.55.11.1247

Bisquerra, R. (2000). Métodos de investigación educativa. Guía práctica. Barcelona: Grupo Editorial CEAC.

Bújez, A. V. (2008). La LOGSE en los Conservatorios Superiores de Música de Andalucía: Una reflexión sobre el currículo. Sevilla: Centro de Documentación Musical de Andalucía.

Chan, M. Y. (2011). The Relationship Between Music Performance Anxiety, Age, Self-Esteem, and Performance Outcomes in Hong Kong Music Students. Tesis doctoral no publicada. Durham, UK: Durham University.

Cid, M. J. (2012). Los conservatorios superiores de Galicia durante la LOGSE. Revista Electrónica de LEEME. Lista Electrónica Europea de Música en la Educación, 29, 1-22.

Clarck, D. B. \& Agras, W. S. (1991). The assessment and treatment of performance anxiety in musicians. American Journal of Psychiatry, 148(5), 598-605. DOI: 10.1176/ajp.148.5.598

Consejería de Educación de la Comunidad de Madrid (2010). Decreto 36/2010, de 2 de junio, del Consejo de Gobierno, por el que se establece el Plan de Estudios para la Comunidad de Madrid, de las enseñanzas artísticas superiores de Grado en Música. Boletín Oficial de la Comunidad de Madrid, 141, 11-253. Disponible en http://www.bocm.es/boletin/CM_Orden_BOCM/2011/06/16/BOCM-20110616-1.PDF

Consejería de Educación de la Comunidad Autónoma de Castilla y León (2011). Decreto 57/2011, de 15 de septiembre, por el que se establece el Plan de Estudios de las Especialidades de Composición, Interpretación y Musicología, de las Enseñanzas Artísticas Superiores de Grado en Música en la Comunidad de Castilla y León. Boletín Oficial de Castilla y León, 183, 72.617-72.823. Disponible en http://bocyl.jcyl.es/boletines/2011/09/21/pdf/BOCYL-D21092011-3.pdf 
Consejería de Educación, Universidades y Empleo de la Región de Murcia (2013). Resolución de 25 de julio de 2013, de la Dirección General de Formación Profesional y Educación de Personas Adultas, por la que se establece para la Comunidad Autónoma de la Región de Murcia el plan de estudios y la ordenación de los estudios superiores de Música, se completan los planes de estudios iniciados en los años académicos 2010-2011 y 2011-2012 y se regula la prueba específica de acceso. Boletín Oficial de la Región de Murcia, 189, 33.079-33.249. Disponible en http://www.borm.es/borm/documento?obj=bol\&id=64375

Dalia, G. (2004). Cómo superar la ansiedad escénica en músicos. Madrid: Mundimúsica Ediciones.

De Vellis, R. F. (2003). Scale development: Theory and applications (2 $2^{\mathrm{a}}$ Ed.). Newbury Park, CA: Sage.

Departamento de Educación, Universidad, Cultura y Deporte del Gobierno de Aragón (2011). Orden de 14 de septiembre de 2011, de la Consejera de Educación, Universidad, Cultura y Deporte, por la que se aprueba el plan de estudios de las enseñanzas artísticas superiores de Grado en Música, Grado en Diseño y Grado en Conservación y Restauración de Bienes Culturales establecidas por la Ley Orgánica 2/2006, de 3 de mayo, de Educación y se implantan dichas enseñanzas en la Comunidad Autónoma de Aragón. Boletín Oficial de Aragón, 195, 20.911-21.139. Disponible en http://www.boa.aragon.es/cgibin/EBOA/BRSCGI?CMD=VEROBJ\&MLKOB=622009780505

Fehm, L. \& Hille, C. (2005). Bühnenangst bei Musikstudierenden. Verhaltenstherapie \& Verhaltensmedizin, 26(2), 199-212.

Fishbein, M., Middlestadt, S. E., Ottati, V., Straus, S. \& Ellis, A. (1988). Medical problems among ICSOM musicians: Overview of a national survey. Medical Problems of Performing Artists, $3(1), 1-8$.

Herrera, L. \& Jorge, G. (2013). Ansiedad escénica musical en estudiantes de flauta travesera. Eufonía. Didáctica de la Música, 57, 43-55.

Jefatura del Estado (2006). Ley Orgánica 2/2006, de 3 de mayo, de Educación. Boletín Oficial del Estado, 106, 17.158-17.207. Disponible en http://www.boe.es/boe/dias/2006/05/04/pdfs/A17158-17207.pdf

Kaspersen, M. \& Gotestam, K. G. (2002). A survey of Music performance anxiety among Norwegian music students. European Journal of Psychiatry, 16(2), 69-80.

Kenny, D. T. \& Ackermann, B. (2009). Optimising physical and mental health in performing musicians. In S. Hallam, I. Cross \& M. Thaut (Eds.), Oxford Handbook of Music Psychology (pp. 390-400). Oxford, UK: Oxford University Press.

Kenny, D. T. \& Osborne, M. (2006). Music performance anxiety: New insights from young musicians. Advances in Cognitive Psychology, 2(2-3), 103-112. DOI: 10.2478/v10053-008$0049-5$

Kenny, D. T., Davis, P. \& Oates, J. (2004). Music performance anxiety and occupational stress amongst opera chorus artists and their relationship with state and trait anxiety and perfectionism. Journal of Anxiety Disorders, 18(6), 757-777. DOI: 10.1016/j.janxdis.2003.09.004

Kenny, D. T. (2008). Music Performance Anxiety. International Handbook of Musicians' Health and Wellbeing. Oxford, UK: Oxford University Press.

Kline, P. (2000). The handbook of psychological testing (2a Ed.). New York: Routledge.

Lee, S. (2002). Musicians' performance anxiety and coping strategies. American Music Teacher, 52(1), 36-39. 
Marchant-Haycox, S. E. \& Wilson, G. D. (1992). Personality and stress in performing artists. Personality and Individual Differences, 13(10), 1061-1068. DOI: 10.1016/01918869(92)90021-G

McAllister, J. A. (2011). Performance Anxiety Amongst Middle School-Aged Wind Instrumentalists as Influenced by Variations in Delivery of Instructional Script Given by Adjudicators During Sight Reading (Unpublished master's thesis in Musical Education Science). Miami, FL: Florida International University.

Ministerio de Educación (2009). Real Decreto 1614/2009, de 26 de octubre, por el que se establece la ordenación de las enseñanzas artísticas superiores reguladas por la Ley Orgánica 2/2006, de 3 de mayo, de Educación. Boletín Oficial del Estado, 259, 89.743-89.752. Disponible en http://www.boe.es/boe/dias/2009/10/27/pdfs/BOE-A-2009-17005.pdf

Ministerio de Educación (2010). Real Decreto 631/2010, de 14 de mayo, por el que se regula el contenido básico de las enseñanzas artísticas superiores de Grado en Música establecidas en la Ley Orgánica 2/2006, de 3 de mayo, de Educación. Boletín Oficial del Estado, 137, 48.48048.500. Disponible en http://www.boe.es/boe/dias/2010/06/05/pdfs/BOE-A-2010-8955.pdf

Orejudo, S., Fernández, T. \& Briz, E. (2012). Resultados de un programa para reducir el miedo y aumentar la autoeficacia para hablar en público en estudiantes universitarios de primer año. Estudios sobre educación, 22, 199-217.

Ortiz Brugués, A. (2008). Music performance anxiety. A review of the literature. Tesis doctoral no publicada. Freiburger Institut für Musikemedizin. Freiburg, Alemania: Albert-LudwigsUniversität Freiburg i. Br.

Osborne, M. S., Kenny, D. T. \& Holsomback, R. (2005). Assessment of MPA in late childhood. International Journal of Stress Management, 12(4), 312-330. DOI: 10.1037/10725245.12.4.312

Petrovich, A. (2004). Performance anxiety: How teachers can help. American Music Teacher, 53(3), 24-27.

Pilatti, A., Godoy, J. \& Brussino, S. (2011). Expectativas hacia el alcohol y consumo de alcohol en niños y adolescentes de Argentina. International Journal of Psychology and Psychological Therapy, 11(1), 13-32.

Renshaw, P. (1999). En defensa de una cultura del aprendizaje en los centros de enseñanzas artísticas. Doce notas preliminaries, 3, 45-48.

Salmon, P. (1990). A psychological perspective on Musical Performance Anxiety: A review of the literature. Medical Problems of Performing Artists, 5(1), 1-11.

Sandgren, M. (2002). Voice, soma, and psyche: A qualitative and quantitative study of opera singers. Medical Problems of Performing Artists, 17(1), 11-21.

Spahn, C. (2006). Lampenfieber und Aufführungsangst bei Musikern - Grundlagen und Therapie. Med Welt, 57(12), 559-563.

Sternbach, D. J. (1995). Musicians: a neglected working population in crisis. In S. L. Sauter \& L. R. Murphy (Eds.), Organizational risk factors for job stress (pp. 283-302, xii, 400). Washington, DC: American Psychological Association.

Sternbach, D. J. (2008). Stress in the lives of music students. Music educator's journal, 94(3), 4248. DOI: $10.1177 / 002743210809400309$

Studer, R., Danusera, B., Hildebrandt, H., Arial, M. \& Gomez, P. (2011). Hyperventilation complaints in music performance anxiety among classical music students. Journal of Psychosomatic Research, 70(6), 557-564. DOI: 10.1016/j.jpsychores.2010.11.004 
Taborsky, C. (2007). Musical Performance Anxiety: A review of literature. Applications of Research in Music Education, 26(1), 15-25. DOI: 10.1177/87551233070260010103

Tamborrino, R. A. (2001). An examination of performance anxiety associated solo performance of college level music majors. Dissertation Abstracts International Section A: Humanities and Social Sciences, 62(5-A), 1636.

Van Kemenade, J. F. L. M., Van Son, M. J. M. \& Van Heesch, N. C. A. (1995). Performance anxiety among professional musicians in symphonic orchestras: a self-reported study. Psycological Reports, 77(2), 555-562. DOI: 10.2466/pr0.1995.77.2.555

Veintimilla, A. (2010). Las enseñanzas musicales en el EESS. In M. L. Vico (Coord.), Las Enseñanzas Artísticas Superiores en el Espacio Europeo de Educación Superior (pp. 115161). Madrid: Ministerio de Educación del Gobierno de España (Ed.).

Wilson, G. D. (1997). Performance anxiety. In D. J. Hargreaves \& A. C. North (Eds.), The social psychology of music (pp. 229-248). Oxford, UK: Oxford University Press.

Yağişhan, N. (2009). A Survey of Music Performance Anxiety among Turkish Music Students. Turkish Journal of Music Education, 2(1), 1-11.

Zarza, F. J., Casanova, O. \& Orejudo, S. (2016a). Ansiedad escénica y constructos psicológicos relacionados. Estudiantes de cinco conservatorios superiores de música españoles. Revista Internacional de Educación Musical, 4, 13-24. DOI: 10.12967/RIEM-2016-4-p013-024

Zarza, F. J., Orejudo, S., Casanova, O. \& Mazas, B. (2016b). Kenny Music Performance Anxiety Inventory: Confirmatory factor analysis of the Spanish version. Psychology of Music, 44(3), 340-352. DOI: 10.1177/0305735614567932

Manuscrito recibido el 7/12/2015 y evaluado anónimamente. Aceptado para su publicación el 13/06/2016. 